\title{
Calibrating Computational Complexity via Definability: The Work of Julia F. Knight
}

Karen Lange

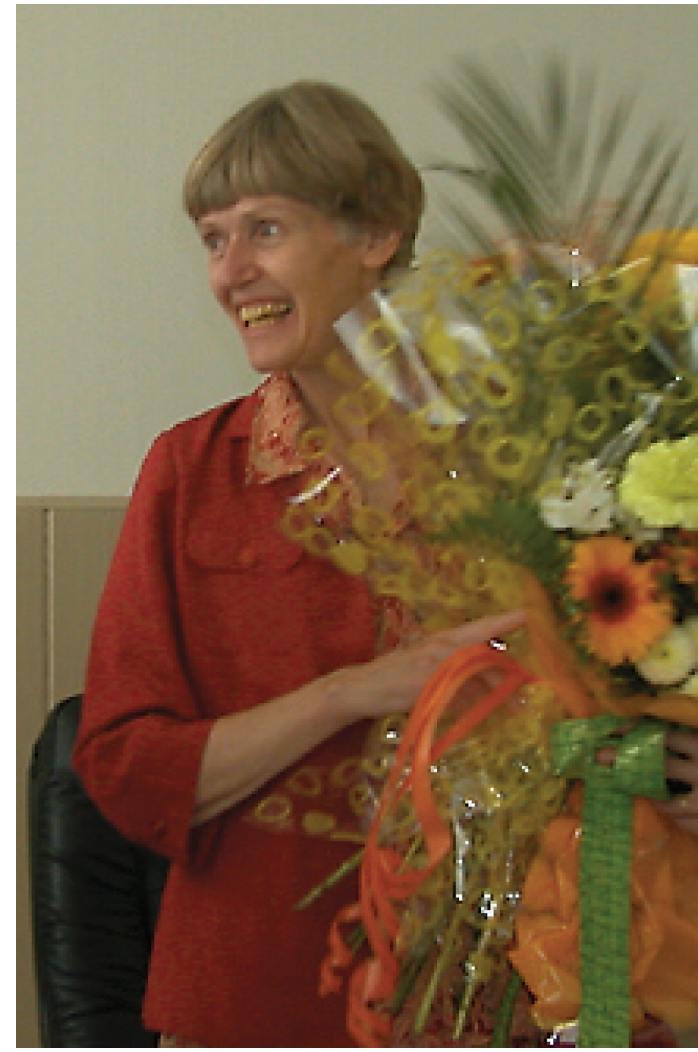

Figure 1. Knight receiving an Honorary Professorship with the Siberian Branch of the Russian Academy of Sciences.

Julia F. Knight, until recently the Charles L. Huisking Professor of Mathematics at the University of Notre Dame, continues to make a significant impact on computability theory and model theory, two subfields of logic, through her research, mentorship, and collaborative approach.

Karen Lange is the Theresa Mall Mullarkey Associate Professor at Wellesley College. Her email address is karen. 1 ange@we11es 1ey. edu.

Communicated by Notices Associate Editor Antonio Montalbán.

For permission to reprint this article, please contact:

reprint-permission@ams.org.
Computability theory provides a framework for calibrating the (idealized) computational content of a mathematical object, whereas model theory is about definabilitywhat can be expressed about a structure in terms of formal syntax. In a nutshell, Knight's work explores how the complexity of syntactic definitions is strongly connected to the computational content of structures. Knight trained as a model theorist at the University of California, Berkeley, under the supervision of Robert Vaught, a key figure in the field's development. After completing her doctorate in 1972 and spending a few years at Penn State, Knight arrived at the University of Notre Dame in 1977, where she has remained throughout her career.

Although she has done some purely model-theoretic work, she was drawn early on into questions about the computational content of structures satisfying the axioms of first-order Peano arithmetic. This work marked the start of her path into computability theory, and her role in shaping and promoting computable structure theory, a subfield of computability that focuses on understanding the computational content encoded by mathematical structures.

The primary goal of this article is to describe Knight's wide-ranging research contributions to logic without assuming background in the field. Her work includes now standard conceptual frameworks that involve an appealing mix of computability-theoretic and model-theoretic ideas and their application to natural examples, some of which are described in her classic text [2] with Chris Ash. In recognition of her research contributions, she was part of the inaugural class of Fellows of the American Mathematical Society in 2012, and she delivered the Association for Symbolic Logic's Gödel Lecture in 2014. She also holds an Honorary Professorship with the Siberian Branch of the Russian Academy of Sciences.

\section{Background}

1.1. Structures, formulas, \& arithmetic. A structure $\mathcal{A}$ consists of an underlying set of elements, known as the domain, and whatever functions or relations on the domain 
that are of interest-the set of symbols $L$ used to denote these functions and relations is called the language of $\mathcal{A}$.

One structure studied extensively in logic is the standard model of arithmetic $\mathbb{N}$, whose domain is $\omega$, the set of natural numbers. ${ }^{1}$ We'll fix the language of $\mathbb{N}$ as $L_{\mathbb{N}}=\{S,+, \cdot,<, 0\}$ so $\mathbb{N}$ comes equipped with the functions giving the successor operation $x \rightarrow x+1$, addition, multiplication, the usual order relation, and a constant for 0 .

Once a language $L$ is fixed, one can define a variety of classes of formal statements using symbols in $L$, logical connectives, variables, and quantifiers. There are natural rules for interpreting the truth or meaning of these statements within a particular structure. In finitary (elementary) first-order logic, formulas are finite in length and variables are intended to range over only the domain of the structure.

For example, for all natural numbers $m$, consider the first-order formula

$$
\theta_{m}=(\exists x) \overbrace{S S \cdots S}^{m} 0<x) .
$$

When interpreted in $\mathbb{N}$, formula $\theta_{m}$ asserts "there is an element in the domain greater than $m$." Formulas, like $\theta_{m}$, in which all variables are bound by a quantifier are called sentences. A sentence is either true or false in a structure, according to natural interpretation rules; certainly $\mathbb{N}$ models $\theta_{m}$, in that $\theta_{m}$ is true in $\mathbb{N}$. Given a collection of sentences $\mathcal{C}$, a structure $\mathcal{A}$ is a model of $\mathcal{C}$ if each of the sentences in $\mathcal{C}$ is true in $\mathcal{A}$. The collection of sentences that are true in $\mathcal{A}$ is called the theory of $\mathcal{A}$, so a structure is a model of its own theory. The theory of $\mathbb{N}$, which contains $\theta_{m}$ for all $m$, is known as true arithmetic (TA).

Nonsentences, such as the subformula of $\theta_{m}$,

$$
\theta_{m}^{\prime}(x)=\overbrace{S S \cdots S}^{m} 0<x)
$$

are valuable for describing subsets of (and relations on) a fixed structure $\mathcal{A}$. If $\delta(x)$ is a formula with one unbound variable $x$, then $\delta(x)$ defines the following subset of $\mathcal{A}$ :

$$
\delta(\mathcal{A}):=\{a \in \text { domain of } \mathcal{A} \mid \delta(a) \text { is true in } \mathcal{A}\} .
$$

Since $\theta_{m}^{\prime}(\mathbb{N})=\{n \in \omega \mid n>m\} \neq \emptyset$ for all natural numbers $m$, the Compactness Theorem for finitary first-order logic guarantees that there are nonstandard models of true arithmetic, ones in which there are elements larger than any natural number. In fact, the standard model $\mathbb{N}$ can be characterized as the only model of $T A$, up to isomorphism, without an element $x$ satisfying the collection of formulas $\{x>\overbrace{S S \cdots S}^{m} 0 \mid m \in \omega\}$. Several of Knight's early papers (e.g., [13]) explored "omitting" collections of formulas in this sense.

\footnotetext{
${ }^{1}$ The symbol $\omega$ comes from viewing the set of natural numbers as the first infinite ordinal.
}

The existence of nonstandard models of $T A$ indicates a limitation in the expressiveness of finitary first-order logic. There is no single finitary first-order formula in $L_{\mathbb{N}}$ stating that some elements are "infinite." But such a formula exists in the infinitary (but still first-order) $\operatorname{logic} \mathcal{L}_{\omega_{1}, \omega}$, which allows formulas with countable conjunctions and disjunctions, namely the (infinitary) formula

$$
(\exists x) \bigwedge_{m \in \omega} \overbrace{S S \cdots S}^{m} 0<x) .
$$

In fact, for each countable structure, there is a single sentence in $\mathcal{L}_{\omega_{1}, \omega}$ that distinguishes the given structure from all others up to isomorphism.

Theorem 1 (Scott Isomorphism Theorem). If $\mathcal{A}$ is a countable structure in a countable language, then there is a sentence in $\mathcal{L}_{\omega_{1}, \omega}$ that is true in just the countable structures isomorphic to $\mathcal{A}$.

The sentence guaranteed by Theorem 1 for a structure $\mathcal{A}$ is called the Scott sentence of $\mathcal{A}$.

Peano arithmetic represents mathematicians' best attempt at giving a useful set of axioms describing the standard model of arithmetic. The axioms of Peano arithmetic are the usual rules for the arithmetic operations and order, along with axioms of the form

$$
[\psi(0) \&(\forall x)(\psi(x) \rightarrow \psi(S x))] \rightarrow(\forall x) \psi(x),
$$

which state that induction holds for any finitary first-order formula $\psi(x)$. The theory of Peano arithmetic, which we denote by $P A$, is the set of all logical consequences of the axioms. As an example, the statement "all nonzero elements have predecessors" is in $P A$ since $P A$ contains the induction axiom in (1) for the formula

$$
x=0 \vee(\exists y)(S y=x)
$$

and can carry out the inductive proof.

Gödel's Incompleteness Theorems imply that $P A$ (and any reasonable axiomatization of $T A$ ) fails to prove all the statements in $T A$, and that $P A$, in fact, can't even prove its own consistency. Further, the inner workings of these results demonstrate that there is no algorithm to determine whether a sentence is in $T A$, or even in $P A$. (There is, however, an algorithm for deciding whether a sentence is an axiom of $P A$.) The relationship between computational complexity and arithmetic goes much deeper and turns out to depend on definability in $\mathbb{N}$.

1.2. Computability. Alan Turing provided the conceptual framework for determining the computational complexity of sets of natural numbers. At the most basic level, a set $A$ of natural numbers is computable if there is an algorithm $^{2}$ that computes the characteristic function $\chi_{A}$ of $A$,

\footnotetext{
${ }^{2}$ By algorithm we really mean a Turing machine, but an informal understanding suffices here by Church's thesis.
} 
and $A$ is computably enumerable (c.e.) if there is an algorithm for listing out the elements of $A$ (not necessarily in order). Note that any computable set $A$ is computably enumerable since we could use $\chi_{A}$ to algorithmically enumerate the elements of $A$ in order.

We can discuss the computability of anything that can be encoded by sets of natural numbers. For example, even though neither $T A$ nor $P A$ is computable, $P A$ is computably enumerable. Indeed, we can algorithmically list all possible deductions from the computable set of axioms for $P A$, by interleaving deductions of varying proof lengths. As we'll see, $T A$ is far from being computably enumerable.

Using the same ideas, we can compare the relative complexity of two subsets of natural numbers $A$ and $B$. The set $A$ is $B$-computable (or computable relative to $B$ ) if there's an algorithm that, given access to the values of $\chi_{B}$, can compute $\chi_{A}$ on any input. In this case, we write $A \leq_{T} B$, and the definition of $B$-computably enumerable is analogous. We describe the computational complexity of a set $A$ by its Turing degree, the equivalence class of sets that are Turing equivalent to $A$ under $\leq_{T}$. In other words, the Turing degree of $A$ is the collection of sets that both compute $A$ and that are $A$-computable.

Just as $P A$ can be computably enumerated, we can effectively enumerate all possible algorithms (written in some specified format) to obtain a computable list of all partial $B$-computable functions on the natural numbers. We'll denote this list by $\left(\Phi_{e}^{B}\right)_{e \in \omega}$. Here $\Phi_{e}^{B}(n)$ is defined only if the $e$ th algorithm reaches an output, i.e., halts, on input $n$ after finitely many steps when given access to $\chi_{B}$. One can use this list to diagonalize outside the class of $B$-computable sets.

Theorem 2. The halting set relative to $B$, defined as

$$
B^{\prime}:=\left\{e \in \omega \mid(\exists s) \Phi_{e}^{B}(e) \text { halts by stage } s,\right.
$$

is a B-computably enumerable set that is not B-computable.

Observe that we can $B$-computably check whether the $e$ th algorithm halts by stage $s$ on input $e$, when given access to $\chi_{B}$. This property can be used to $B$-computably enumerate the elements of $B^{\prime}$, making $B^{\prime}$ c.e. relative to $B$. Furthermore, note that the definition of $B^{\prime}$ is an existential one in terms of a $B$-computable property. The set $B^{\prime}$ is often called the jump of $B$ because the complexity of $B^{\prime}$ "jumps above" that of $B$, i.e., $B^{\prime}>_{T} B$. Hence, the set $\emptyset^{\prime}$ is a natural example of a c.e. set that is not computable. We can continue to take jumps to obtain

$$
\emptyset<_{T} \emptyset^{\prime}<_{T} \emptyset^{\prime \prime}=\emptyset^{(2)}<_{T} \emptyset^{\prime \prime \prime}=\emptyset^{(3)}<_{T} \cdots .
$$

These sets end up serving as an important measuring stick for computational power in the arithmetical hierarchy.

1.2.1. The arithmetical hierarchy. Even with its expressive limitations, finitary first-order logic can define many subsets of $\mathbb{N}$. Matiyasevich's negative resolution of Hilbert's
Tenth Problem implies that all computably enumerable sets are definable in $\mathbb{N}$ using relatively simple formulas. Hilbert's Tenth Problem asks whether there's an algorithm for determining whether a given Diophantine equation, one of the form $p(\bar{x}, \bar{y})=0$ where $p(\bar{x}, \bar{y}) \in \mathbb{Z}[\bar{x}, \bar{y}]$, has a solution in the integers. Building on the work of Robinson, Davis, and Putnam, Matiyasevich provided a characterization of the solution sets of Diophantine equations.

Theorem 3 (MRDP Theorem). Let $S$ be a subset of $\mathbb{Z}^{j}$. The set $S$ is computably enumerable if and only if

$$
S=\left\{\bar{n} \in \mathbb{Z}^{j} \mid\left(\exists \bar{y} \in \mathbb{Z}^{k}\right)[p(\bar{n}, \bar{y})=0\}\right.
$$

for some $p(\bar{x}, \bar{y}) \in \mathbb{Z}[\bar{x}, \bar{y}]$.

Theorem 3 together with the existence of noncomputable c.e. sets like $\emptyset^{\prime}$ ensure that no algorithm of the kind Hilbert hoped for exists. Moreover, since the integers can be represented simply in terms of natural numbers, Theorem 3 implies that all c.e. sets are definable in $\mathbb{N}$ by finitary formulas whose only quantifiers are existential. Such formulas are known as $\Sigma_{1}^{0}$ formulas.

This relationship between computational complexity and definability extends to all arithmetical sets, those that are definable in $\mathbb{N}$ by a formula in finitary first-order logic. The arithmetical hierarchy classifies these definable subsets by the syntactic complexity of the formulas defining them. A formula (in prenex normal form) is $\Sigma_{n+1}^{0}$ if it starts with an existential quantifier followed by at most $n$ alternations between blocks of existential and universal quantifiers, and then a quantifier-free formula. ${ }^{3}$ The $\Pi_{n+1}^{0}$ formulas are similarly defined, with the initial quantifier being universal. Finally, a set of natural numbers is described as $\Sigma_{n}^{0}$ (respectively, $\Pi_{n}^{0}$ ) if it is defined in $\mathbb{N}$ by a formula at that level and $\Delta_{n}^{0}$ if it is both $\Sigma_{n}^{0}$ and $\Pi_{n}^{0}$. Sometimes one wants to describe a property in terms of some parameter set $B$ of natural numbers. One can make the same definitions all relative to the given $B$, so we can talk about $\Sigma_{n}^{0}(B)$ or $\Pi_{n}^{0}(B)$ subsets of natural numbers.

The arithmetical hierarchy is tightly aligned with the computational complexity of subsets of $\mathbb{N}$, when measured against the sequence $\left(\emptyset^{(n)}\right)_{n \in \omega}$. Post's Theorem explains this relationship ${ }^{4}$ and shows that the hierarchy does not collapse.

Theorem 4 (Post). Let $A$ be a set of natural numbers.

1. $A$ is $\Delta_{n+1}^{0}$ if and only if $A \leq_{T} \emptyset^{(n)}$.

2. $A$ is $\Sigma_{n+1}^{0}$ if and only if $A$ is $\emptyset^{(n)}$-computably enumerable. Hence, $A$ is definable by a $\Sigma_{1}^{0}$ formula in $\mathbb{N}$ if and only if $A$ is c.e.

\footnotetext{
${ }^{3}$ The superscript 0 indicates the formulas are first order.

${ }^{4}$ The formulation here of the arithmetical hierarchy relies on Theorem 3, a much later result than Post's.
} 
3. $\emptyset^{(n)}$ is $\Sigma_{n}^{0}$-complete for all $n>0$ in that

(a) $\emptyset^{(n)}$ is $\Sigma_{n}^{0}$, and

(b) for every $\Sigma_{n}^{0}$ set $B$, there is a one-to-one computable function $f$ such that $x \in B$ if and only if $f(x) \in \emptyset^{(n)}$.

\section{Complexity of Structures}

While Knight was at Penn State, Mark Nadel recruited her to join him at the University of Notre Dame in 1977. Like Knight, Nadel was a model theorist studying models of arithmetic from that field's perspective, but their joint efforts began to require some methods from computability. At the same time, others had become interested in the computational content of models of $P A$ and $T A$, including Carl Jockusch, a computability theorist at Urbana-Champaign, and David Marker and his advisor Angus Macintyre, two model theorists then at Yale. Working on the computational content of models of arithmetic solidified Knight's path into computability theory.

Here we've begun blurring the idea of a structure with a specific encoding of one. To encode a (countable) structure $\mathcal{A}$, one labels its domain with constants $\left(c_{i}\right)_{i \in \omega}$ and considers the collection $D(\mathcal{A})$ of quantifier-free facts about $\mathcal{A}$ in terms of these constants. The collection $D(\mathcal{A})$ is known as the atomic diagram of $\mathcal{A}$. Observe that the collection $D(\mathcal{A})$ here depends on the labeling of the domain of $\mathcal{A}$. Each possible encoding of $D(\mathcal{A})$ is called a presentation or copy of $\mathcal{A}$, whose computational content can be measured. From here on out, we will identify a structure $\mathcal{A}$ with a particular encoding of $D(\mathcal{A})$. So, the computational content of $\mathcal{A}$ is measured by the Turing degree of its particular encoding, the equivalence class of all sets equivalent to this encoding under $\leq_{T}$.

2.1. Degrees of models of arithmetic. Though Tennenbaum had earlier shown that there are no computable nonstandard models of $P A$ (and hence $T A$ ), Jockusch and Soare had demonstrated in 1972 that "low" nonstandard models of $P A$ exist. A low set $X$ is one that is almost computable in the sense that its jump $X^{\prime}$ is as small as possible, i.e., $X^{\prime} \leq_{T} \emptyset^{\prime}$.

The situation for $T A$ is quite different; this theory and its nonstandard models are quite complex. By its construction, $T A$ can uniformly compute whether a given formula holds in $\mathbb{N}$. Since Post's Theorem states that $\emptyset^{(n)}$ is defined by a $\Sigma_{n}^{0}$ formula, any set $X$ computing $T A$ can uniformly compute $\emptyset^{(n)}$ for all $n$.

As for models of $T A$, a result of Fefferman (which Knight and Nadel later generalized) shows that if $\mathcal{A}$ is a nonstandard model of $T A$, then $\operatorname{deg}(\mathcal{A})>_{T} \emptyset^{(n)}$ for all $n$. Knight conjectured that Fefferman's result was the only limitation on the complexity of nonstandard models of $T A$, i.e., that, for each $X>_{T} \emptyset^{(n)}$ for all $n$, there would be a model of $T A$ having the same degree as $X$. In 1984, Knight, together with Lachlan and Soare, proved this conjecture to be false. (See [12] for references in this section.)

2.2. Degrees of presentations. The above work motivated Knight and others to study different presentations of a given countable structure. The degrees of these presentations can vary widely, making the following definition natural.

Definition 1. The degree spectrum of a countable structure $\mathcal{A}$ is

$$
\operatorname{DgSp}(\mathcal{A})=\{\operatorname{degree}(\mathcal{B}) \mid \mathcal{B} \cong \mathcal{A}\} .
$$

In 1982, Marker proved that the degree spectrum of a model $\mathcal{A}$ of $P A$ is upwards closed, in that, given a degree $\mathbf{d}$ Turing above a presentation of $\mathcal{A}$, there is another copy of $\mathcal{A}$ of exactly degree $\mathbf{d}$. Macintyre asked what other kinds of structures, beyond models of arithmetic, have this property. Knight's answer [14], obtained while visiting Yale in the fall of 1982, is a classic result of computable structure theory.

Theorem 5 (Knight). Let $\mathcal{A}$ be a countable structure. The following are equivalent:

1. The degree spectrum $\operatorname{DgSp}(\mathcal{A})$ is upward closed.

2. $\mathcal{A}$ is automorphically nontrivial, in that there is no finite subset $S$ of the domain of $\mathcal{A}$ such that all permutations fixing $S$ are in fact automorphisms.

Her proof shows how automorphically nontrivial structures are rich enough to allow (and are necessary) for the coding of additional computational information. Knight continued to study questions involving the degree spectra of structures, e.g., what sets are computable in all copies of a given structure, while visiting Jockusch at UrbanaChampaign for her sabbatical in 1984-85 and throughout her career.

\section{Complexity Between Copies}

Knight met Chris Ash, a computability theorist at Monash University who would become a close collaborator, during her 1984-85 sabbatical. Their highly regarded book [2] describes their shared interests (see this text for all references in this section). The book was not intended to be jointly authored, but Knight completed the project after Ash's untimely death in 1995. The book addresses three kinds of problems regarding the complexity between and within particular copies of a fixed structure. So as to give some concrete motivation, ${ }^{5}$ we'll first state their most basic incarnations.

Problem 1. Given a computable structure $\mathcal{A}$ and a relation $R$ on $\mathcal{A}$, what syntactic conditions ensure that the image of $R$ is c.e. under all isomorphisms from $\mathcal{A}$ to another computable copy?

\footnotetext{
${ }^{5}$ This exposition is inspired by Rod Downey's excellent Math Review of [2].
} 
A relation on a structure $\mathcal{A}$ is called intrinsically c.e. if it has the property in Problem 1 . Let $(\omega,<)$ be the standard linear order on the natural numbers. The relation " $x<y$ but $y$ is not the successor of $x$ " in $(\omega,<)$ is intrinsically c.e.; given a computable copy of $(\omega,<)$, one can computably enumerate the pairs of domain elements $(x, y)$ whenever another element $z$ is placed between $x$ and $y$. Observe that this relation is defined by the $\Sigma_{1}^{0}$ formula $(\exists z)(x<z \& z<y)$, suggesting the relevance of definability. On the other hand, the successor relation itself turns out not to be intrinsically c.e., even though it's computable in the standard model $(\omega,<)$.

Problem 2. Given a computable structure $\mathcal{A}$, what syntactic conditions ensure that every computable copy $\mathcal{B}$ of $\mathcal{A}$ is computably isomorphic to $\mathcal{A}$ ?

Structures $\mathcal{A}$ with the property described in Problem 2 are called computably categorical. For example, the usual linear order on the rationals $(\mathbb{Q},<)$ is computably categorical; the construction of an isomorphism between two countable dense linear orders via the usual back and forth argument is an algorithmic process. But, $(\omega,<)$ is not computably categorical, since that would imply this structure's successor relation would be intrinsically c.e., which, as we stated above, is false.

Problem 3. Given a computable structure $\mathcal{A}$, what syntactic conditions ensure that, for every computable copy $\mathcal{B}$ of $\mathcal{A}$, every isomorphism from $\mathcal{B}$ to $\mathcal{A}$ is computable?

Though $(\omega,<)$ is not computably categorical (and hence does not have the desired property in Problem 3), all isomorphisms between its computable copies are $\Delta_{2}^{0}$. Indeed, any isomorphism between copies of $(\omega,<)$ must match up the least elements, and then their successors, and then those elements' successors, etc. Combining this process with the fact that the least element and successor relations are both $\Delta_{2}^{0}$-definable gives the desired result.

In earlier work, others explored Problems 1, 2, and 3 as stated, in general and in specific kinds of structures, e.g., Goncharov and independently Remmel proved that the computably categorical linear orders are those with only finitely many successor pairs. However, the answers to the original problems are typically quite complicated. As we'll see, the problems have more elegant solutions when the copies range over all copies of a fixed structure, rather than only those of a given complexity. For example, a computable structure $\mathcal{A}$ is called relatively computably categorical if for every copy $\mathcal{B}$ of $\mathcal{A}$, there is a $\mathcal{B}$-computable isomorphism from $\mathcal{B}$ to $\mathcal{A}$. Changing the problems in this way is one approach to relativizing. Another approach is to relativize the other objects under consideration (e.g., replace "computable isomorphism" with " $\Delta_{2}^{0}$-isomorphism" as done in the example after Problem 3). Knight and Ash, often with others, focused on relativizing these problems according to both approaches, to the hyperarithmetical hierarchy, an extension of the arithmetical hierarchy.

3.1. Hyperarithmetical hierarchy. The measuring stick of the arithmetical hierarchy is the sequence $\left(\emptyset^{(n)}\right)_{n \in \omega}$, in the sense that a set of natural numbers is arithmetical if it is computable from $\emptyset^{(n)}$ for some $n$. We can extend this measuring stick by continuing to take jumps along the infinite ordinals, as long as the ordinals (and hence this process) remain sufficiently effective:

$$
\emptyset<_{T} \emptyset^{\prime}<_{T} \emptyset^{(2)}<_{T} \cdots<_{T} \emptyset^{(\omega)}<_{T} \emptyset^{(\omega+1)}<_{T} \cdots .
$$

Such ordinals are those that are order-isomorphic to a computable well-ordering. These computable ordinals form a countable initial segment of all ordinals; the notation $\omega_{1}^{C K}$ denotes the least noncomputable ordinal.

A subset of $\mathbb{N}$ is hyperarithmetical if it is computable from $\emptyset^{(\alpha)}$ for some computable ordinal $\alpha$. True arithmetic provides a natural example; the degree of $T A$ is that of $\emptyset^{(\omega)}$.

3.1.1. Computable infinitary formulas. Just like the arithmetical sets, the hyperarithmetical sets can be described in terms of definability. Here we use computable infinitary formulas, formulas from $L_{\omega_{1}, \omega}$ whose countable conjunctions and disjunctions are computably enumerable. As in the finitary first-order setting, formula complexity is measured by alternation of quantifiers. The quantifier-free finitary first-order formulas are deemed both $\Sigma_{0}^{c}$ and $\Pi_{0}^{c}$. Then, for any computable ordinal $\alpha$, a formula is $\Sigma_{\alpha}^{c}$ if it is essentially a (possibly infinite) c.e. disjunction of formulas $(\exists \bar{u}) \psi(\bar{u})$, where the $\psi(\bar{x})$ are $\Pi_{\beta}^{c}$ formulas for varying $\beta<\alpha$. Analogously, a formula is $\Pi_{\alpha}^{c}$ if it is a c.e. conjunction of formulas $(\forall \bar{u}) \psi(\bar{u})$, where the $\psi(\bar{x})$ are $\Sigma_{\beta}^{c}$ formulas for different $\beta<\alpha$.

The next theorem, which follows from results related to Theorem 8 , provides evidence that the computable infinitary formulas are the "right" logic for the hyperarithmetical setting.

Theorem 6. A set is hyperarithmetical if and only if it is definable in $\mathbb{N}$ by a computable infinitary formula. Moreover, if a set $A$ is hyperarithmetical, then for any computable $\alpha \geq \omega$,

- $A$ is definable by a $\Sigma_{\alpha}^{c}$ and a $\Pi_{\alpha}^{c}$ formula if and only if $A$ is $\emptyset^{(\alpha)}$-computable;

- $A$ is definable by a $\Sigma_{\alpha}^{c}$ formula if and only if $A$ is $\emptyset^{(\alpha)}$. computably enumerable.

We remark that the $\Sigma_{n}^{c}$ and $\Pi_{n}^{c}$ formulas do not define anything in $\mathbb{N}$ beyond what their finitary analogues could. As before, we declare a set of natural numbers $\Sigma_{\alpha}^{0}$ if it is definable by a $\Sigma_{\alpha}^{c}$ formula; $\Pi_{\alpha}^{0}$ or $\Delta_{\alpha}^{0}$ subsets are defined similarly.

3.1.2. Connection to analytic hierarchy. The hyperarithmetical sets can also be defined in terms of the first level of the analytical hierarchy, a classification of subsets of $\mathbb{N}$ in terms of their definability in second-order arithmetic, a logic that 
allows variables ranging over not just natural numbers but also sets of natural numbers. Here quantifiers ranging over sets (and their alternations) are what drive complexity. A formula of this kind is $\Sigma_{1}^{1}$ (resp., $\Pi_{1}^{1}$ ) if it has only existential (resp., universal) set quantifiers. ${ }^{6}$ The hyperarithmetical sets coincide with the $\Delta_{1}^{1}$ sets, those that are definable in $\mathbb{N}$ by both $\Sigma_{1}^{1}$ and $\Pi_{1}^{1}$ formulas.

Computable infinitary formulas are also the "right" logic for understanding computable structures (and hyperarithmetical ones too). Most crucially, for any computable ordinal $\alpha$, a $\Sigma_{\alpha}^{c}$ formula defines a $\Sigma_{\alpha}^{0}$ subset of the domain of a computable structure (and an analogous result holds for $\Pi_{\alpha}^{c}$ ones). They also have a compactness theorem akin to the one for finitary first-order logic, that can even provide computable models.

Theorem 7 (Barwise-Kreisel Compactness). If $\Gamma$ is a $\Pi_{1}^{1}$ set of computable infinitary sentences and every hyperarithmetical subset of $\Gamma$ has a (computable) model, then $\Gamma$ has a (computable) model.

One can apply Barwise-Kreisel Compactness to show that computable infinitary formulas are capable of distinguishing between nonisomorphic computable structures.

Corollary 1. If $\mathcal{A}$ and $\mathcal{B}$ are computable (or hyperarithmetical structures) satisfying the same computable infinitary sentences, then $\mathcal{A} \cong \mathcal{B}$.

3.2. Results on the relativizations. By their nature, Problems 1,2 , and 3 in the introduction to $\$ 3$ require understanding how computational power is connected to definability. In fact, the resolution of the full relativization of Problem 1 is purely about definability.

Theorem 8 (Ash, Knight, Manasse, \& Slaman, independently Chisholm). Let $R$ be a relation on a computable structure $\mathcal{A}$. The following are equivalent:

1. $R$ is definable in $\mathcal{A}$ by a $\Sigma_{\alpha}^{c}$ formula referencing a finite tuple of parameters from $\mathcal{A}$.

2. For any $\mathcal{B} \cong \mathcal{A}$, the image of $R$ in $\mathcal{B}$ is $\Sigma_{\alpha}^{0}(\mathcal{B})$.

A relation $R$ on a computable structure $\mathcal{A}$ satisfying statement 2 of Theorem 8 is called relatively intrinsically $\Sigma_{\alpha}^{0}$ on $\mathcal{A}$. Recall that the adverb "relatively" here indicates that $\mathcal{B}$ is ranging over all copies of $\mathcal{A}$, not just the computable ones. This aspect of the definition reflects the first approach to relativizing described at the end of the introduction to $\$ 3$. Considering images of relations that are $\Sigma_{\alpha}^{0}(\mathcal{B})$, rather than just those that are $\Sigma_{1}^{0}(\mathcal{B})$, reflects the second approach mentioned. Because there are no restrictions on the complexity of $\mathcal{B}$, this "relative" definition requires only that the image of $R$ in $\mathcal{B}$ be $\Sigma_{\alpha}^{0}(\mathcal{B})$, rather than plainly $\Sigma_{\alpha}^{0}$.

\footnotetext{
${ }^{6}$ The subscript " 1 " indicates that there are element variables and set variables.
}

The syntactic conditions in Theorem 8 were first identified in the computable setting by Ash and Anil Nerode and then Ash's student Ewan Barker. Though those results came first, Theorem 8 can be stated and proven more elegantly, suggesting that combining "relative" approaches leads to stronger results. Progress on Problems 2 and 3 evolved similarly.

The solutions to Problems 2 and 3 (whether relativized or not) involve the ability to effectively enumerate formulas that define certain parts of the structure. Goncharov showed the solution to Problem 2 involves a special kind of Scott family, an important tool in the proof of the Scott Isomorphism Theorem.

Definition 2. A Scott family for a structure $\mathcal{A}$ is a collection of formulas $\mathcal{F}$ in $\mathcal{L}_{\omega_{1}, \omega}$ (in terms of some fixed parameters) so that

- each tuple in $\mathcal{A}$ satisfies some formula in $\mathcal{F}$, and

- if two tuples in $\mathcal{A}$ satisfy the same formula in $\mathcal{F}$, then there's an automorphism of $\mathcal{A}$ taking one to the other.

Theorem 9 (Ash, Knight, Manasse, \& Slaman, independently Chisholm). For a computable structure $\mathcal{A}$, the following are equivalent:

1. $\mathcal{A}$ has a c.e. Scott family consisting of $\Sigma_{\alpha}^{c}$ formulas in terms of a fixed tuple of parameters.

2. For any $\mathcal{B} \cong \mathcal{A}$, there is a $\Delta_{\alpha}^{0}(\mathcal{B})$-computable isomorphism from $\mathcal{A}$ to $\mathcal{B}$, i.e., $\mathcal{A}$ is relatively $\Delta_{\alpha}^{0}$-categorical.

Showing that the given computational feature implies the desired definability is the difficult direction in both Theorems 8 and 9. The proofs of these portions use forcing, a powerful technique of mathematical logic. Forcing here involves building a "generic" copy $\mathcal{B}$ of the given structure $\mathcal{A}$, whose construction "forces" decisions about statements describing the computational properties of $\mathcal{B}$. Since $\mathcal{B}$ is, in fact, a copy of $\mathcal{A}$ and by assumption $\mathcal{B}$ has the specified computational feature, this fact must be forced at some point in the construction, allowing one to extract the desired definability statement.

3.3. Priority arguments. While Theorems 8 and 9 rely on forcing, the solutions of the original three problems use finite injury priority constructions, a fundamental proof technique in computability theory. Friedberg and independently Muchnick first used this method to build a c.e. set $B$ strictly between $\emptyset$ and $\emptyset^{\prime}$. A priority construction is a stageby-stage process for making an object according to a list of "requirements" that together ensure desired properties. Typically the requirements make use of the list of all partial $B$-computable functions $\left(\Phi_{e}^{B}\right)_{e \in \omega}$. For example, a single requirement $R_{e}$ in the Friedberg-Muchnick Theorem aims to ensure that the eth partial computable function $\Phi_{e}^{\emptyset}$ does not compute $B$. Satisfying requirement $R_{e}$ for all $e$ guarantees that $B>_{T} \emptyset$. 
The strategies for satisfying distinct requirements may conflict. In fact, satisfying one requirement may "injure" or undo the satisfaction of another. Hence, an ordering is put on the requirements to make clear at any stage which requirement has priority to act. In a finite injury construction, like that of the Friedberg-Muchnick Theorem and those for the original three problems above, each requirement is injured only finitely often. Though these constructions are computable, knowing when requirements are permanently satisfied is $\emptyset^{\prime}$-computable.

Shoenfield and Sacks independently invented infinite injury constructions, to obtain theorems like Sacks' result that the degrees of c.e. sets are dense. Determining how requirements are satisfied in these constructions requires increasing amounts of power. For example, Harrington used his "workers" method to obtain a nonstandard model of arithmetic that was arithmetical but whose theory was not. Determining how requirements are met in this construction requires $\emptyset^{(\omega)}$. Knight and others tried with some success to employ Harrington's approach in other settings, but priority constructions need increasingly intricate mechanisms as their requirements involve more complicated information. Coming up with, understanding, and verifying these constructions was becoming unmanageable.

To ameliorate this situation, Ash developed a black box approach. His "metatheorem" guarantees that if certain effectiveness conditions are satisfied, then an associated infinite injury priority construction will succeed. Knight joined forces with Ash and others to refine this method, develop new variations, e.g., [15], and explore applications, including ones related to the three problems.

\section{Complexity of Classes of Structures}

Though well aware of his work before, Knight met Sergei Goncharov (Novosibirsk State University) at an Association for Symbolic Logic conference in Leeds in the summer of 1997 before joining him at a conference in Kazan. There Goncharov gave a talk describing a variety of effective classification results for specific classes of computable structures (e.g., computable linear orders) and calling for similar results for other natural classes. At the talk, Richard Shore asked Goncharov what would make him give up on finding an effective classification of a particular class. Shore's question led Knight and Goncharov to explore three known approaches to effective classification and to identify a satisfying answer in [9]. This work generated a new wave of interest in both the theory of effective classification and its application to natural classes, from graphs, to fields, to groups.

Let $\mathcal{K}$ be a class of structures that is closed under isomorphism (e.g., fields of characteristic zero). Goncharov and Knight aimed to understand characterizations and classifications of $\mathcal{K}^{c}$, the set of computable presentations of elements in $\mathcal{K}$. For them, an effective characterization of $\mathcal{K}$ is a way to understand the full diversity of structures in $\mathcal{K}^{c}$ (possibly with repetition), and an effective classification of $\mathcal{K}$ is a method that also distinguishes between isomorphism types within $\mathcal{K}^{c}$. (See [4] for a more comprehensive view of these approaches and for the references in this section.)

\subsection{Three approaches.}

4.1.1. Enumerations. Classification in mathematics often takes the form of a list of all distinct possibilities, up to a fixed notion of equivalence, e.g., the classification of finite simple groups. This idea motivates the first approach.

Definition 3. An enumeration of $\mathcal{K}^{c}$ is a sequence $\left(\mathcal{C}_{n}\right)_{n \in \omega}$ of presentations in $\mathcal{K}^{c}$ in which every $\mathcal{A} \in \mathcal{K}^{c}$ is isomorphic to some $\mathcal{C}_{n}$.

A Friedberg enumeration of $\mathcal{K}^{c}$ is an enumeration of $\mathcal{K}^{c}$ in which the isomorphism types of the $\mathcal{C}_{n}$ are distinct.

Thus, an enumeration characterizes the computable structures in $\mathcal{K}$, and a Friedberg enumeration classifies them. The moniker "Friedberg" has its origin in Friedberg's classic result providing a computable enumeration of all c.e. sets without repetition. Goncharov and Knight viewed an enumeration, whether Friedberg or not, as being effective if the enumeration is hyperarithmetical.

4.1.2. Computable infinitary descriptions \& Scott rank. The second approach is syntactic, relating to Scott sentences and Scott families. In this approach, an effective characterization of $\mathcal{K}$ is a computable infinitary definition of $\mathcal{K}^{c}$, if any exists. Describing effective classification in this approach requires more effort.

In a standard proof of the Scott Isomorphism Theorem, one first shows that there is a Scott family for the given structure $\mathcal{A}$ and an ordinal $\alpha$ in which the definitions of all orbits of tuples in $\mathcal{A}$ are $\Pi_{\alpha}^{0}$. The least such ordinal $\alpha$ for which such a Scott family exists can be used to define the Scott rank of $\mathcal{A}$, a measure of syntactic complexity of certain automorphisms. The Scott ranks of computable structures have nice features.

\section{Theorem 10 (Nadel). Let $\mathcal{A}$ be a computable structure.}

1. The Scott rank of $\mathcal{A}$ is at most $\omega_{1}^{C K}+1$.

2. $\mathcal{A}$ has computable Scott rank (so less than $\omega_{1}^{C K}$ ) if and only if $\mathcal{A}$ has a computable infinitary Scott sentence.

Turning to a class $\mathcal{K}$ of structures, Goncharov and Knight deemed $\mathcal{K}$ effectively classifiable if there is a computable ordinal bound on the Scott ranks of the elements of $\mathcal{K}^{c}$. Such a bound implies that there is a computable ordinal $\alpha$ such that structures in $\mathcal{K}^{c}$ all have $\Pi_{\alpha}^{c}$ Scott sentences, which distinguish these structures up to isomorphism. Hence, this bound on Scott rank is a proxy for effective classification. 
4.1.3. Index sets \& the isomorphism problem. The third approach measures the computational content of sets involving the "names" of presentations in $\mathcal{K}^{c}$.

Definition 4. The index set of $\mathcal{K}$ is the set $I(\mathcal{K})$ of indices $e$ of functions $\Phi_{e}^{\emptyset}$ that encode a presentation in $\mathcal{K}^{c}$.

The isomorphism problem of $\mathcal{K}$ is the set $E(\mathcal{K})$ of ordered pairs $\left(e, e^{\prime}\right)$ so that $e, e^{\prime} \in I(\mathcal{K})$ and the structures computed by $\Phi_{e}^{\emptyset}$ and $\Phi_{e^{\prime}}^{\emptyset}$ are isomorphic.

Here Goncharov and Knight viewed $\mathcal{K}$ as having an effective characterization if $I(\mathcal{K})$ is hyperarithmetical and an effective classification if $E(\mathcal{K})$ is.

4.1.4. Relationships between approaches. At the level of determining whether a class $\mathcal{K}$ is effectively classifiable, all three approaches turn out to be equivalent, under the assumption that $\mathcal{K}$ can be characterized by a computable infinitary sentence. The complexity of such a syntactic description of $\mathcal{K}$ gives an upper bound on the computational content of $I(\mathcal{K})$-e.g., if $\mathcal{K}^{c}$ is described by a $\Pi_{3}^{c}$ statement, then $I(\mathcal{K})$ is $\Pi_{3}^{0}$. Even better, Goncharov and Knight showed that $\mathcal{K}$ has a computable infinitary characterization if and only if $I(\mathcal{K})$ is hyperarithmetical. Building on Goncharov and Knight's work, Calvert and Knight stated the following result.

Theorem 11. If $I(\mathcal{K})$ is hyperarithmetical, then the following are equivalent:

1. $\mathcal{K}$ has a hyperarithmetical Friedberg enumeration.

2. There is a computable ordinal $\alpha$ such that any two members of $\mathcal{K}^{c}$ satisfying the same $\Pi_{\alpha}^{c}$ sentences are isomorphic.

3. $E(\mathcal{K})$ is hyperarithmetical.

Theorem 11 provides a robust test for determining whether a class is effectively classifiable. Applying Theorem 11, one can show natural classes like vector spaces over a fixed infinite computable field, algebraically closed fields of fixed characteristic, and archimedean real closed fields are effectively classifiable whereas classes like undirected graphs, fields of fixed characteristic, real closed fields, and linear orders are not.

4.2. Finer-grained complexity. A hallmark of Knight's work is the marriage of theory-building and application, and Knight and her collaborators have used all three approaches to obtain finer-grained information about specific effectively classifiable classes. Exact complexity calculations make use of the structural properties of the class and often employ results of classical (noneffective) mathematics. In this section, we highlight some of this work, beginning with Knight and her collaborators work on free groups in [5]. All references in $\$ 4.2 .1$ can be found there.

4.2.1. Free groups. In 1945, Tarski asked whether all free groups with at least two, but not infinitely many, generators are elementarily equivalent, i.e., satisfy the same finitary first-order statements in the language of groups. In
2006, Sela and independently Kharlampovich and Myasnikov proved the answer is positive-finitary first-order logic is unable to distinguish between finitely generated free groups of rank at least two. Since all countable free groups have computable presentations, they are distinguished from one another by computable infinitary formulas by Corollary 1 . Hence, it's natural to search for computable infinitary descriptions of these groups. Any such description of these groups puts an upper bound on the computational content of their index sets. A description of a group $G$ is optimal if $I(G)$ is $m$-complete at the level of the description.

Definition 5. Let $G$ be a countable group. The index set $I(G)$ is $m$-complete at a given complexity level if

1. $I(G)$ has the given complexity, and

2. for every set $X$ of the given complexity, there is a uniformly computable sequence of groups $\left(G_{n}\right)_{n \in \omega}$ such that $n \in X$ if and only if $G_{n} \cong G$.

Let $F_{n}$ denote the free group of rank $n$, i.e., one having a basis of size $n$, and let $F_{\infty}$ be the free group of countably infinite rank. Recall that a basis for a free group is a generating set for the group in terms of which there is no nontrivial expression of the identity.

Knight and her collaborators, including some of her then current and former students, found optimal descriptions for $F_{n}, F_{\infty}$, and related classes of groups in [5]. They showed that $F_{\infty}$ has a $\Pi_{4}^{c}$ description, which McCoy and Wallbaum, a subset of the authors, showed was optimal. Simply determining whether a presentation of a group is free is quite complicated, so the full team also looked for descriptions that distinguish one specific free group from other free groups. The group $F_{\infty}$ has an optimal $\Pi_{3}^{c}$ description of this latter kind.

When applied to the class of all groups, however, the $\Pi_{3}^{c}$ description of $F_{\infty}$ no longer characterizes $F_{\infty}$, since it may describe groups that are locally free but not free. A group is locally free if every finitely generated subgroup is a free group. An example of a locally free but not free group that satisfies the $\Pi_{3}^{c}$ description is the abelian group generated by $\left\{b_{n} \mid n \in \omega\right\}$ such that $b_{n+1}^{2}=b_{n}$. To obtain the $\Pi_{4}^{c}$ description that picks out exactly $F_{\infty}$ amongst all groups, the authors needed to describe tuples of elements that can be part of a basis. They relied on Nielsen transformations on collections of words, which are analogous in flavor to row reductions on matrices. Just as there is reduced echelon form for matrices, there is an $\mathrm{N}$-reduced form on words. Given a tuple of words, $N$-reduced form can be used to determine whether the tuple could be part of a basis or not, if applied to a set of basis elements. Moreover, the processes involved in making this determination are computable.

Interestingly, the optimal descriptions of the groups and classes are not always the "natural" ones. Knight and 
her collaborators note that an obvious description of $F_{n}$ states that there is an $n$-tuple, having no nontrivial relationships, so that every other group element can be written as a word on this tuple. This description is $\Sigma_{3}^{c}$. When they went to show this description was optimal, they became stuck, motivating them to search for (and find) a simpler but less expected description. Thus, computable structure theory helps to pinpoint wherein lies the true complexity within a problem.

4.2.2. Friedberg enumerations. Though the three approaches agree on whether a class has an effective classification or not, the exact complexities may differ between approaches. For example, computable Friedberg enumerations exist for vector spaces over a fixed infinite computable field and algebraically closed fields of fixed characteristic, but Calvert showed that the isomorphism problem $E(\mathcal{K})$ for these classes is $m$-complete $\Pi_{3}^{0}$, i.e., is $\Pi_{3}^{0}$ and computably encodes any $\Pi_{3}^{0}$ set. The complexities are relatively low since (countable) vector spaces and algebraically closed fields have simple invariants in terms of dimension and transcendence degree.

Equivalence structures, ones whose domain $\omega$ is equipped with an equivalence relation, also have relatively simple invariants in terms of the number of equivalence classes of a given size, but they are slightly more syntactically difficult to describe. Indeed, the computable bound on the Scott rank of equivalence structures is higher than that of those on vector spaces and algebraically closed fields, and the isomorphism problem $E(\mathcal{K})$ is a level more complex at $m$-complete $\Pi_{4}^{0}$.

Determining whether the class of equivalence structures has a computable Friedberg enumeration proved challenging. Goncharov and Knight were able to show that the class of all equivalence structures with infinitely many infinite classes has a computable Friedberg enumeration, but conjectured that the full class did not. Others proved related but not definitive existence and nonexistence results. However, Goncharov and Knight's conjecture was falseDowney, Melnikov, and Ng provided a Friedberg enumeration of all equivalence structures in 2017. (See [7] for the attributions in \$4.2.2.)

4.2.3. Results when $\mathcal{K}$ is nonclassifiable. If a class $\mathcal{K}$ is not effectively classifiable, Theorems 10 and 11 imply that the bound on the Scott ranks of elements of $\mathcal{K}^{c}$ must be "high," either $\omega_{1}^{C K}$ or $\omega_{1}^{C K}+1$. Knight and her collaborators have a large body of work on computable structures of high Scott rank. Prior to their work, it was well known that, for any computable ordinal $\alpha$, a computable structure of Scott rank $\alpha$ exists. Researchers also knew of computable examples of rank $\omega_{1}^{C K}+1$-the most famous being the Harrison order. Even though $\omega_{1}^{C K}$ has no computable presentation by definition, Harrison proved that there is a computable linear order whose order type consists of an initial segment of type $\omega_{1}^{C K}$ followed by densely many copies of $\omega_{1}^{C K}$. This order has Scott rank $\omega_{1}^{C K}+1$. That left the question of whether there exist computable structures of Scott rank $\omega_{1}^{C K}$. In 1981 Makkai proved that an arithmetical structure of rank $\omega_{1}^{C K}$ exists. Knight and Jessica Millar showed that this structure can be made computable.

Theorem 12 (Knight \& Millar). There is a computable structure of Scott rank $\omega_{1}^{C K}$.

With Calvert, Goncharov, and Millar, Knight showed that there are computable structures of Scott rank $\omega_{1}^{C K}$ in natural classes such as undirected graphs, trees, fields of any characteristic, and linear orders. Other classes cannot sustain such examples, e.g., abelian $p$-groups by a result of Barwise. The computable infinitary theories of these examples are all $\aleph_{0}$-categorical in that they have exactly one countable model up to isomorphism. Millar and Sacks produced a nonhyperarithmetical example of Scott rank $\omega_{1}^{C K}$ whose theory is not $\aleph_{0}$-categorical, but asked whether the example could be made computable. In 2018, Harrison-Trainor, Igusa, and Knight produced such an example (see [10] for the references in this section).

Knight, together with a large team, also studied the class of high rank structures using the tools described earlier. They showed that the index set of the class of high rank structures is as complicated as possible, specifically $\Sigma_{1}^{1}$-complete [3].

4.3. Another approach to classification. In the early 2000s, Alexander Kechris suggested to Knight that an approach to classification from descriptive set theory might have a natural effective analogue. In descriptive set theory, as above, structures are identified with their atomic diagrams, which in turn are identified with their encodings as elements of Cantor space $2^{\omega}$, the set of infinite sequences of 0 's and 1's. The class of all structures in a fixed countable language forms a Polish space, a separable and complete metric space. For a class $\mathcal{K}$ of structures closed under isomorphism, one can show $\mathcal{K}$ is Borel if and only if $\mathcal{K}$ is definable by a sentence in $L_{\omega_{1}, \omega}$.

A coarse measure of classification complexity in this setting is whether the isomorphism relation on $\mathcal{K}$ is Borel. The following approach gives more information.

Definition 6 (Friedman \& Stanley). Let $\mathcal{K}$ and $\mathcal{K}^{\prime}$ be classes of structures that are closed under isomorphism. A Borel embedding of $\mathcal{K}$ into $\mathcal{K}^{\prime}$ is a Borel function $F$ from $\mathcal{K}$ to $\mathcal{K}^{\prime}$ such that

$$
\mathcal{A} \cong \mathcal{A}^{\prime} \Longleftrightarrow F(\mathcal{A}) \cong F\left(\mathcal{A}^{\prime}\right)
$$

A class is Borel complete if it is Borel and every class is Borel embeddable in it.

Examples of Borel complete classes include linear orders, trees, and undirected graphs. But Borel reducibility 
does not allow for distinctions between classes having countably many isomorphism types.

Following Kechris' suggestion, Knight and her then students Calvert, Cummings, and Miller introduced computable and Turing computable embeddings, as two ways to distinguish between classes with countably many isomorphism types. Here these embeddings directly translate statements about a structure in $\mathcal{K}$ into statements about a structure in $\mathcal{K}^{\prime}$. In [16], Knight and a different group of students proved a "Pull-back Theorem" that guarantees that syntactic invariants for the codomain class can be transformed into syntactic invariants in the domain class in both approaches (see this citation for all prior references in $\$ 4.3)$.

Knight and others [8] also explored an analogous kind of computable embedding introduced by Friedman and Fokina that maps elements of $I(\mathcal{K})$ to elements of $I\left(\mathcal{K}^{\prime}\right)$. Knight and her various collaborators have applied these frameworks to a wide variety of classes and compared their behavior to each other and to related approaches.

\section{Further Contributions}

Though we've described many important threads of Knight's work, there are others. We briefly mention a few more.

5.1. Computable model theory. Knight would continue to explore ideas connected to those in \$2.1. If a theory $T$ (such as $T A$ ) has a computable model, the set of $\Sigma_{n}^{0}$ statements in $T$ is $\Sigma_{n}^{0}$ uniformly in $n$. Andrews, Lempp, and Schweber called such theories Solovay theories (see their paper [1] for all references in this section). Earlier, Knight and Solovay proved that a set computes a copy of some model of every Solovay theory if and only if it computes a nonstandard model of $T A$. However, when the Solovay theory $T$ has exactly one countable model up to isomorphism, Knight (building on work of Lerman and Schmerl) obtained a better result. In this case, she showed that the only countable model of $T$ has a $\emptyset^{\prime}$-computable copy.

More recently, Knight and Andrews studied Solovay theories that are strongly minimal, an important class of theories in model-theoretic stability. A minimal structure is one in which each subset defined by a finitary first-order formula is finite or cofinite. In other words, the only definable subsets of the domain are those that are definable using equality and inequality alone. A theory is strongly minimal if all its models are minimal. Many natural theories are strongly minimal, e.g., those of vector spaces over $\mathbb{Q}$ and algebraically closed fields of a fixed characteristic.

Knight and Andrews proved that all countable models of a strongly minimal Solovay theory have a $\emptyset^{(3)}$. computable copy and asked whether $\emptyset^{(3)}$ could be replaced by $\emptyset^{(2)}$. Andrews, Lempp, and Schweber recently proved the answer is no, by characterizing the sets $X$ that compute a copy of every countable model of a strongly minimal Solovay theory. These are precisely the high sets over $\emptyset^{(2)}$, i.e., sets $X$ satisfying $X \geq_{T} \emptyset^{\prime \prime}$ and $X^{\prime} \geq_{T} \emptyset^{(4)}$.

5.2. Recursive saturation, integer parts, \& Hahn fields. Recursive saturation is a property of abundance, in that structures with this property have elements that satisfy any reasonable, describable property. Knight extensively studied this important model-theoretic notion and its relationship to models of arithmetic.

Definition 7. A structure is recursively saturated if any computable collection of finitary first-order formulas that is consistent with the structure is modeled by some elements in the structure.

As one example of her work in this area, Knight, D'Aquino, and Starchenko studied integer parts of real closed fields [6]. (See this citation for references in \$5.2.) A real closed field is an ordered field that has the same theory as $\mathbb{R}$, i.e., one in which square roots of positive elements and roots of odd degree polynomials exist. An integer part $I$ of a real closed field $R$ is a discrete ordered ring in which every element of $R$ is within distance of 1 of exactly one element of $I$. So, an integer part $I$ sits inside $R$ just as $\mathbb{Z}$ does in $\mathbb{R}$. Mourgues and Ressayre proved every real closed field has at least one integer part. While archimedean real closed fields have $\mathbb{Z}$ as their unique integer part, nonarchimedean ones may have many.

Shepherdson showed that a discrete ordered ring $I$ is an integer part of a real closed field if and only if $I$ is a model of a subset of $P A$ called IOpen, in which the induction axioms are limited to quantifier-free formulas. The following theorem illuminates what happens when an integer part $I$ is a model of full $P A$.

Theorem 13 (D'Aquino, Knight, Starchenko).

1. If a real closed field $R$ has an integer part $I$ that is a nonstandard model of $P A$, then $R$ is recursively saturated. Hence, in particular, the real closure of I is recursively saturated.

2. If $R$ is a countable recursively saturated real closed field, then $R$ has an integer part $I$ such that $R$ is the real closure of $I$ and $I$ is a nonstandard model of $P A$.

To prove a given real closed field $R$ has an integer part, Mourgues and Ressayre showed that $R$ can be specially embedded in a Hahn field, a field of generalized power series in which the series have ordinal lengths. Understanding this process led Knight and her collaborators to study the complexity of root-taking in Hahn fields and the simpler setting of fields of Puiseux series, when these fields are algebraically closed and of characteristic zero. They proved that Newton's method for finding roots over a field of Puiseux series is fully computable, and uniformly so for nonconstant polynomials (see [11] for all citations in this 


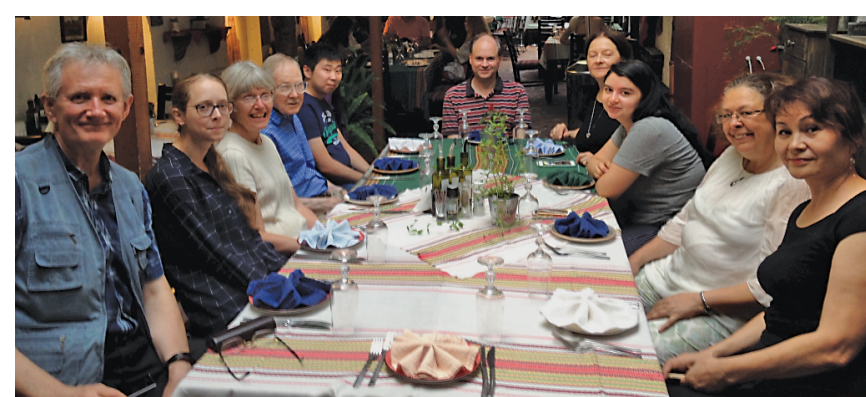

Figure 2. Julia and her husband Bill with collaborators and friends in Bulgaria.

paragraph). In the Hahn field setting, Knight and Lange found sharp bounds on the ordinal lengths of roots in terms of the lengths of the polynomial's coefficients. These length bounds seem to play an important role in understanding the exact complexity of the root-taking process in Hahn fields, an area of ongoing work.

5.3. o-minimality. Knight also played a role in the development of o-minimality, an important generalization of strong minimality formalized by Anand Pillay and Charles Steinhorn. An o-minimal structure is one with an order in which each subset of the domain defined by a finitary firstorder formula is a finite union of points and intervals, i.e., those that are definable using only equality and the order alone. In analogy with the strongly minimal setting, a theory is $o$-minimal if all its models are $o$-minimal. The canonical example of an $o$-minimal theory is the theory of $\mathbb{R}$ as an ordered field (which, in contrast with $T A$, is computable). The study of $o$-minimality has allowed for the generalization of results about real algebraic geometry and semialgebraic sets. In particular, Knight, Pillay, and Steinhorn [17] proved a fundamental cell decomposition theorem for $o$-minimal theories.

\section{Epilogue}

Mathematicians can shape their discipline not only through their intellectual contributions but also their approach to the mathematics community. Knight collaborates extensively with others at all career stages, and she generously acknowledges others' ideas (even when rescuing a germ of a good idea from an ill-conceived proposal).

She supports collaboration and communication in other ways as well. Early in her career, she cofounded the Midwest Model Theory seminar with John Baldwin at Illinois-Chicago. This seminar continues to run today and inspired analogous seminar series in computability in the midwest and northeast. A lack of communication between the East and West led to substantial research duplication in computability during and after the Cold War. Knight has been a major proponent of East/West collaboration, facilitating funding and travel in both directions for logicians. Her efforts along with those of others, particularly
Steffen Lempp who started an NSF East/West collaboration grant in the late 1990s that Knight now administers, have helped to integrate these research communities and propel the field forward. She continues to steward the logic community as the immediate past president of the Association for Symbolic Logic.

Knight is an exemplary mentor and advocate for earlycareer mathematicians (including but not limited to her eighteen doctoral students), greeting new faces at conferences, securing funding, and making valuable introductions. These efforts are not limited to logicians either. She served as Director of Graduate Studies at Notre Dame for many years and was awarded the university's 2007 James A. Burns, C.S.C., Graduate School Award for distinction in graduate education.

ACKNOWLEDGMENTS. The author is very grateful for Knight's ongoing mentorship, collaboration, and friendship, and her assistance in putting together this article. She also thanks the anonymous referees for their thoughtful suggestions.

\section{References}

[1] Uri Andrews, Steffen Lempp, and Noah Schweber, Building models of strongly minimal theories, Adv. Math. 386 (2021), Paper No. 107802, 25, DOI 10.1016/j.aim.2021.107802. MR4266747

[2] C. J. Ash and J. Knight, Computable structures and the hyperarithmetical hierarchy, Studies in Logic and the Foundations of Mathematics, vol. 144, North-Holland Publishing Co., Amsterdam, 2000. MR1767842

[3] W. Calvert, E. Fokina, S. S. Goncharov, J. F. Knight, O. Kudinov, A. S. Morozov, and V. Puzarenko, Index sets for classes of high rank structures, J. Symbolic Logic 72 (2007), no. 4, 1418-1432, DOI 10.2178/jsl/1203350796. MR2371215

[4] Wesley Calvert and Julia F. Knight, Classification from a computable viewpoint, Bull. Symbolic Logic 12 (2006), no. 2, 191-218. MR2223921

[5] J. Carson, V. Harizanov, J. Knight, K. Lange, C. McCoy, A. Morozov, S. Quinn, C. Safranski, and J. Wallbaum, Describing free groups, Trans. Amer. Math. Soc. 364 (2012), no. 11, 5715-5728, DOI 10.1090/S0002-9947 2012-05456-0. MR2946928

[6] P. D'Aquino, J. F. Knight, and S. Starchenko, Real closed fields and models of Peano arithmetic, J. Symbolic Logic 75 (2010), no. 1, 1-11, DOI $10.2178 /$ jsl/1264433906 MR2605879

[7] Rodney G. Downey, Alexander G. Melnikov, and Keng Meng $\mathrm{Ng}$, A Friedberg enumeration of equivalence structures, J. Math. Log. 17 (2017), no. 2, 1750008, 28, DOI 10.1142/S0219061317500088 MR3730564 


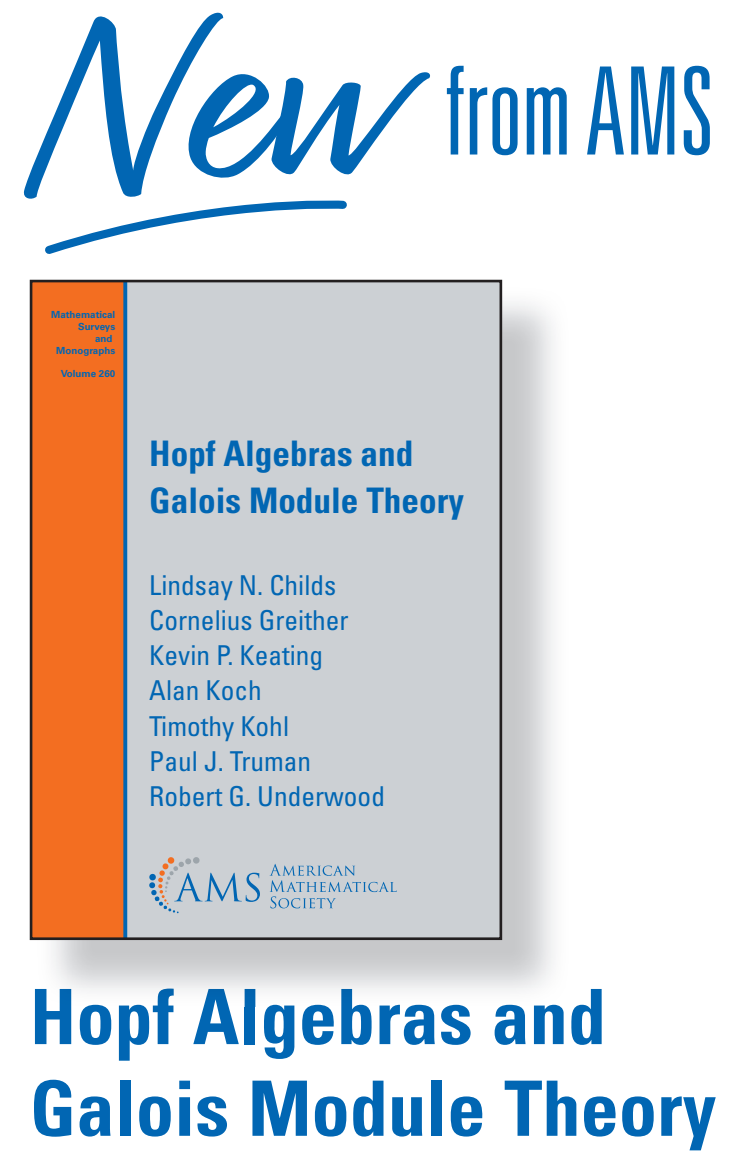

Lindsay N. Childs, University at Albany, $N Y$, Cornelius Greither, Universität der Bundeswehr München, Neubiberg, Germany, Kevin P. Keating, University of Florida, Gainesville, FL, Alan Koch, Agnes Scott College, Decatur, GA, Timothy Kohl, Boston University, MA, Paul J. Truman, Keele University, Staffordshire, United Kingdom, and Robert G. Underwood, Auburn University at Montgomery, $A L$

This book surveys the state of the art in HopfGalois theory and Hopf-Galois module theory and can be viewed as a sequel to the first author's book, Taming Wild Extensions: Hopf Algebras and Local Galois Module Theory.

Mathematical Surveys and Monographs, Volume 260; 2021; 311 pages; Softcover; ISBN: 978-1-47046516-2; List US\$125; AMS members US\$100; MAA members US\$112.50; Order code SURV/260

\section{Visit|bookstore.ams.org}

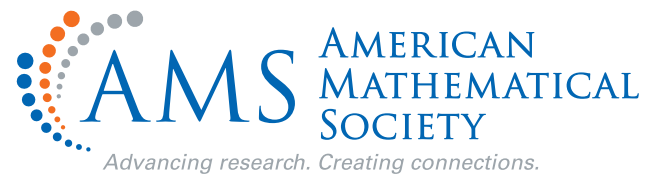

[8] Ekaterina B. Fokina, Sy-David Friedman, Valentina Harizanov, Julia F. Knight, Charles McCoy, and Antonio Montalbán, Isomorphism relations on computable structures, J. Symbolic Logic 77 (2012), no. 1, 122-132, DOI 10.2178/jsl/1327068695. MR2951633

[9] S. S. Goncharov and Dzh. Naĭt, Computable structure and antistructure theorems (Russian, with Russian summary), Algebra Logika 41 (2002), no. 6, 639-681, 757, DOI 10.1023/A:1021758312697; English transl., Algebra Logic 41 (2002), no. 6, 351-373. MR1967769

[10] Matthew Harrison-Trainor, Gregory Igusa, and Julia F. Knight, Some new computable structures of high rank, Proc. Amer. Math. Soc. 146 (2018), no. 7, 3097-3109, DOI 10.1090/proc/13967. MR3787370

[11] J. Knight, K. Lange, and D. R. Solomon, Roots of polynomials in fields of generalized power series, Proceedings for Aspects of Computation, World Scientific, to appear.

[12] Julia Knight, Alistair H. Lachlan, and Robert I. Soare, Two theorems on degrees of models of true arithmetic, J. Symbolic Logic 49 (1984), no. 2, 425-436, DOI 10.2307/2274174 MR745370

[13] Julia F. Knight, Omitting types in set theory and arithmetic, J. Symbolic Logic 41 (1976), no. 1, 25-32, DOI 10.2307/2272941, MR406793

[14] Julia F. Knight, Degrees coded in jumps of orderings, J. Symbolic Logic 51 (1986), no. 4, 1034-1042. MR865929

[15] Julia F. Knight, Requirement systems, J. Symbolic Logic 60 (1995), no. 1, 222-245, DOI 10.2307/2275519. MR1324511

[16] Julia F. Knight, Sara Miller, and M. Vanden Boom, Turing computable embeddings, J. Symbolic Logic 72 (2007), no. 3, 901-918, DOI 10.2178/jsl/1191333847. MR2354906

[17] Julia F. Knight, Anand Pillay, and Charles Steinhorn, Definable sets in ordered structures. II, Trans. Amer. Math. Soc. 295 (1986), no. 2, 593-605, DOI 10.2307/2000053 MR833698

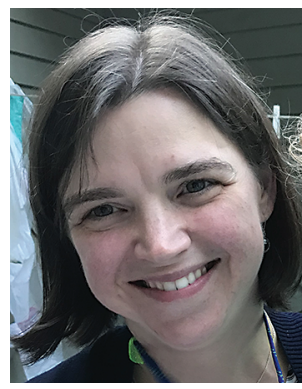

Karen Lange

Credits

Figure 1 is courtesy of Wesley Calvert.

Figure 2 is courtesy of Charlie McCoy.

Photo of Karen Lange is courtesy of Darlene Howland. 